\title{
Force Sensing Technique for Capacitive Touch Panel
}

\author{
Shuo Gao, Arokia Nathan \\ Hetero-Genesys Laboratory, Department of Engineering, University of Cambridge, \\ Cambridge, CB3 OFA, U.K. \\ E-mail:sg690@cam.ac.uk, an299@cam.ac.uk
}

\begin{abstract}
A force sensing technique is presented for capacitive touch panels. By measuring force induced stress, force touch can be detected even when the panel deflection is very small. In addition to that, image based down-sampling methods are proposed to reduce power consumption and boost readout speed of touch panel.
\end{abstract}

\section{Author Keywords}

force-sensing; capacitive touch panel; down-sampling; power reduction.

\section{Introduction}

Force sensing for capacitive touch screens is a desired function which expands touch detection from $2 \mathrm{D}$ to $3 \mathrm{D}$, and is recently commercially provided by Apple Inc. The technique for interpreting force level relies on the deflection caused by the force of the touch [1]. However, this becomes challenging when the force touch occurs at the edge of the screen, resulting in poor force sensitivity. To solve this issue, this paper employs a force sensing layer, in which the stress caused by force can be measured. The amount of generated stress is proportional to the strength of the applied force, thus the force level can be obtained. To investigate the mechanical property of the force sensing layer based touch panel, COMSOL simulation results were studied. The stack-up and parameters are depicted in Fig. 1.

As additional circuitry is needed to interpret force touch signal, system's power consumption is increased, resulting in a short battery life time. To solve this issue, down-sampling method is utilized in this paper. From [2], it is learned that a finger touch normally affects $3 \times 3$ touch sensors, when a force touch occurs, even more electrodes are influenced. If we treat a touch related frame as a picture, then each electrode contributes to a pixel value. The pixel intensities of the pixels within touch affected area follow a certain distribution (e.g. Gaussian distribution), which is widely used in sub-pixel interpolation. Thus, by only measuring a portion of them, the values of un-sampled pixels can be estimated. As the reconstructed touch position may be shifted compared to the original one, a regional scan is performed around the reconstructed touch position to retrieve accurate touch information. By employing down-sampling technique, low power consumption and fast readout can be achieved at the same time.

\section{Touch Panel Displacement and Stress Analysis}

Touch screen panel could be modeled as a thin flat panel. The boundary condition is between a simple supported case and a fully clamped case. The theoretical analysis of the former case is available in [3]. However, little literature of the latter case could be found. In fact, the real boundary condition is close to the latter case.

In this section, simulation results from COMSOL are used to analyse the displacement of touch panel and stress on the force

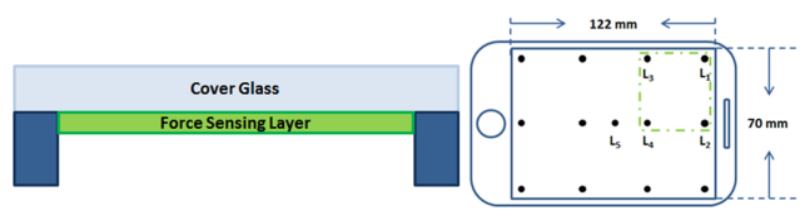

(a)

(b)

Figure 1. (a) Cross section of proposed force sensing technique. Electrodes are on and underneath the force sensing layer. As electrodes are much thinner compared to the other layers, they are not depicted here. (b) Top view of proposed touch panel and investigated touch locations.

sensing layer for the fully clamped case. Uniform forces over a small concentric circle of radius $1 \mathrm{~mm}$ were applied at locations 1 to 5 , with different strength levels. The results for locations 1 to 3 are illustrated below in Fig. 2, since these three locations are near the edges of the touch panel.

It can be observed that the displacements are at the $\mu \mathrm{m}$ scale even below-, indicating that the change in capacitance is very small, which can be easily affected by noise. In contrast, the stress change to the force sensing layer is significant.

\section{Down-sampling and Reconstruction Technique}

The number of touch sensors affected by a finger touch mainly depends on three factors. They are finger size, sensor size and sensor spacing. The diameter of a finger is around $7 \mathrm{~mm}$ to $15 \mathrm{~mm}$, and a typical electrode size and spacing are both $5 \mathrm{~mm}$, considering the fringing field effect, at least $3 \times 3$ electrodes will be affected, normally more electrodes are involved [2]. In nowadays, to achieve more accurate touch detection, smaller electrode and spacing sizes are used. In our experiments, the electrode size is $3 \mathrm{~mm}$ and the spacing distance is $2 \mathrm{~mm}$, thus more electrodes are affected as depicted in Fig. 3 (a). Below we analyze the property of finger touch from pixel level (the touch based frame is treated as a picture, each electrode intersection is assumed as a pixel) and frame level.

From the view of pixel level, the pixels affected by a finger touch signal follow a certain distribution (e.g. Gaussian distribution). This is widely used in subpixel interpolation to estimate the accurate touch position. The popularly used distributions are linear and Gaussian distributions. In this paper, we assume the pixels follow linear distribution. From the view of frame level, compared to the noise spikes, the touch affected region has a spatial low frequency property. In contrast, compared to the spatial low-frequency noise, the touch signal normally has a high value.

When half column-and-row electrodes are used, four scenarios can happen to touch signals, as conceptually illustrated in Fig. 4. Here different colors represent pixel intensities induced by touch events. 


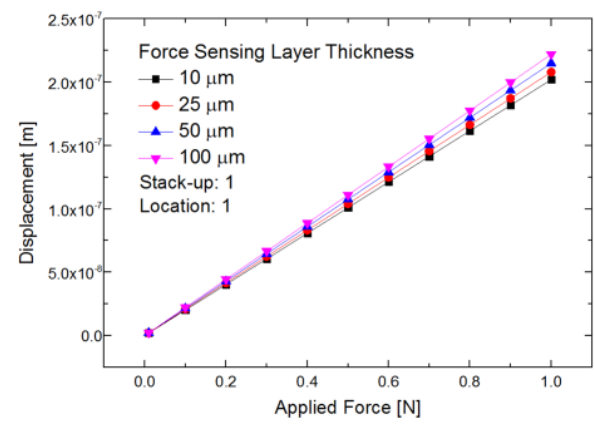

(a)

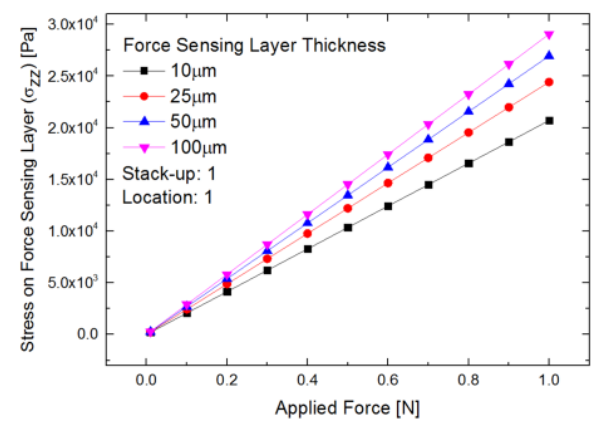

(d)

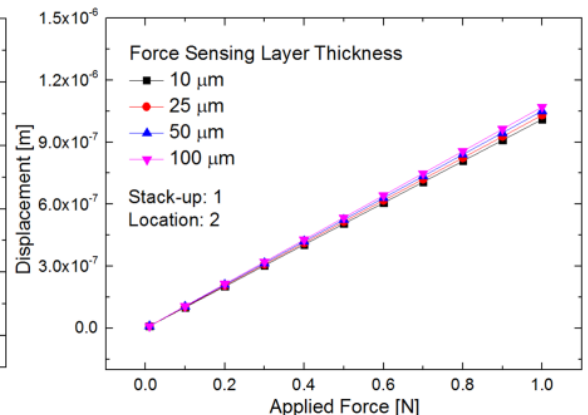

(b)

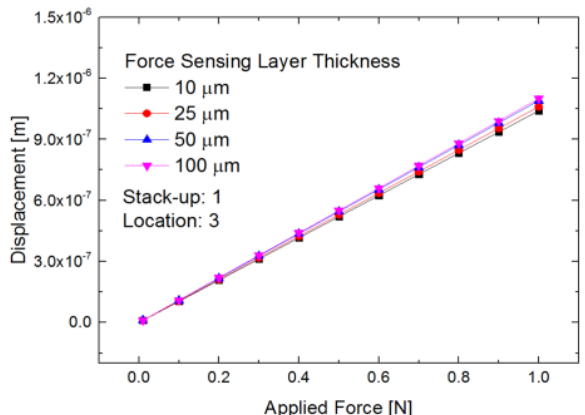

(c)

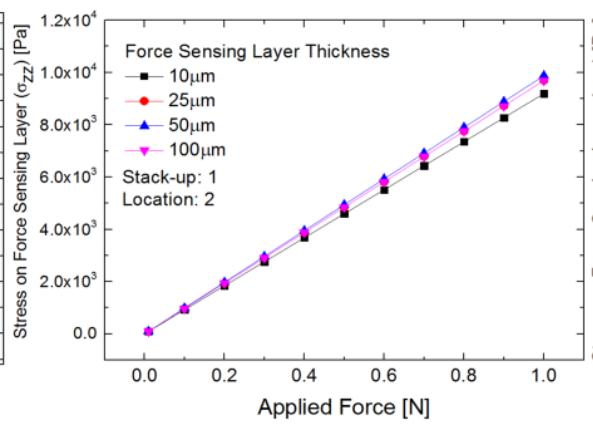

(e)

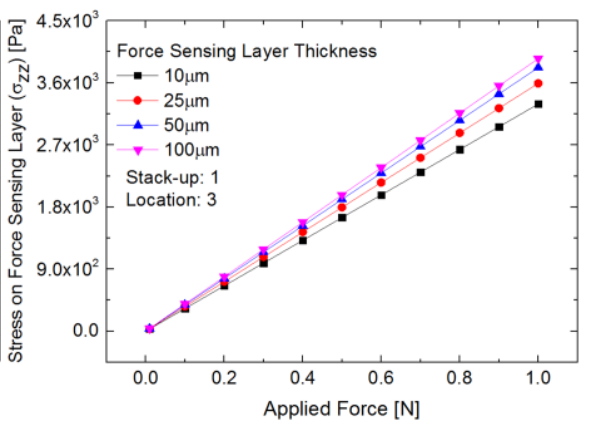

(f)

Figure 2. Displacement (a) - (c) and stress (d) - (f) results for stackup 1, at locations 1 to 3 , with applied force from 0.1 to $1 \mathrm{~N}$.

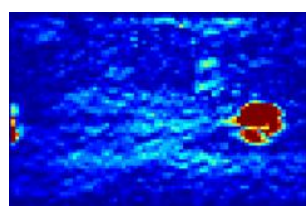

(a)

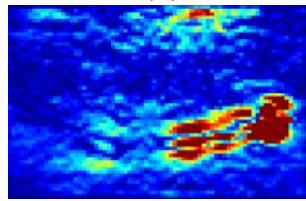

(c)

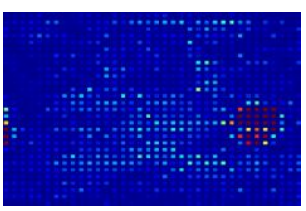

(b)

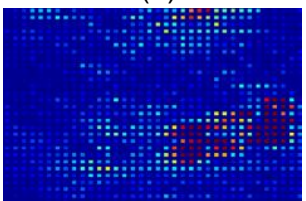

(d)
Figure 3. The touch related images before and after down-sampling. (a) and (c) are raw images of one and multi-touch signals; (b) and (d) are corresponding downsampled signals.

The origin finger touch mainly affects $3 \times 3$ pixels, the touch position pixel has the highest intensity, and pixels with the same distance from the touch position are assumed to have similar values, thus denoted by the same color. By analyzing the downsampled frames, we learn that the sampled pixels are surrounded by the blank pixels (zeros inserted and denoted by black color), the touch position pixel has a high probability $(75 \%)$ to be not sampled. Hence, the aiming of the algorithm is to keep the sampled values and estimate the blank areas. As mentioned in section II, we assume the pixels within the touch area follow linear distribution. Thus, we have

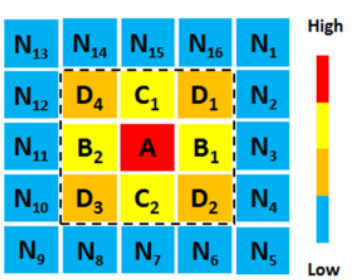

(a)

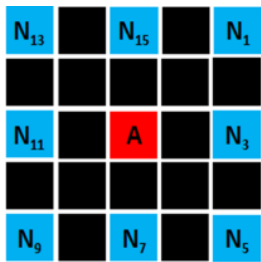

(b)

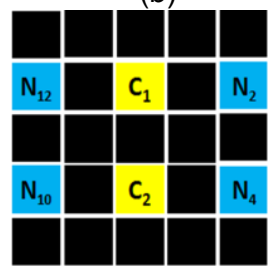

(d)

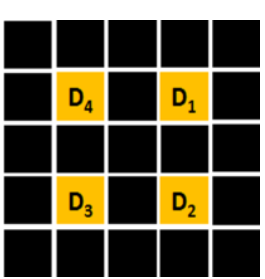

(c)

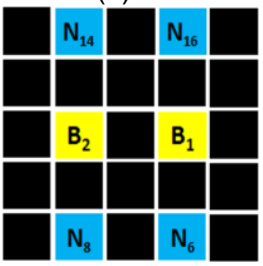

(e)
Figure 4. (a) Original touch signal; (b)-(e) four scenarios of down-sampled touch signal.

$$
B_{1}=B_{2}=C_{1}=C_{2}=\alpha A ; \quad D_{1}=D_{2}=D_{3}=D_{4}=\beta A
$$




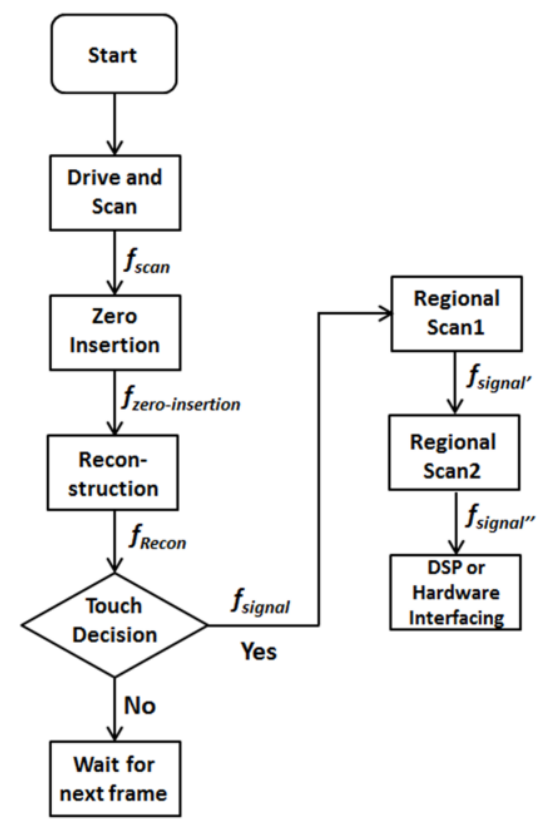

Figure 5. Flowchart of proposed down-sampling and reconstruction algorithm.

The values of factors $\alpha$ and $\beta$ are within the range of 0 to 1 , and highly depend on the touch panel structure, electrode pattern, finger property and touch position. However, we can still approximately estimate their values based on the touch signal property analysis. In this paper, $\alpha$ is 0.9 and $\beta$ is 0.8 . Therefore the reconstruction algorithm is

$$
\begin{aligned}
& V_{(x, y)}^{\prime}=V_{(x, y)}+\frac{\alpha}{2}\left(V_{(x, y+1)}+V_{(x, y-1)}\right)+\frac{\alpha}{2}\left(V_{(x+1, y)}+V_{(x-1, y)}\right) \\
& +\frac{\beta}{4}\left(V_{(x-1, y-1)}+V_{(x-1, y+1)}+V_{(x+1, y-1)}+V_{(x+1, y+1)}\right)
\end{aligned}
$$

where $(x, y)$ represents the pixel location, $V_{(x, y)}$ and $V_{(x, y)}^{\prime}$ are the values of the original pixel value and the reconstructed pixel value at the location $(x, y)$ respectively.

As descripted in Fig. 5, after only driving and scanning half rows and columns $\left(f_{\text {scan }}\right)$, zeros are inserted to the down-sampled touch frame to recover the resolution $\left(f_{\text {zero-insertion }}\right)$. Then the pixel intensity distribution based reconstruction algorithm is applied to reconstruct the original touch position. On the positive touch decision, a regional scan is performed around the reconstructed touch position to obtain the accurate one $\left(f_{\text {signal }}\right)$. A subsequent regional scan is optionally performed to retrieve accurate and detailed touch information $\left(f_{\text {signal }},\right)$ for specific purposes, e.g. sub-pixel interpolation. Finally the accurate touch information is processed for various purposes.

Four reconstruction results and corresponding original touch signal are illustrated in Fig. 6. As stated above, the reconstructed touch position may be shifted, thus a regional scan is needed to be performed. The radius of the regional scan depends on the reconstruction quality. Here we choose the worst reconstruction and analyze its cross section to decide the regional scan range. As shown in Fig. 7, by using one order Gaussian curve to fit the

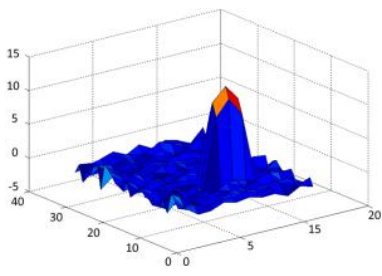

(a)

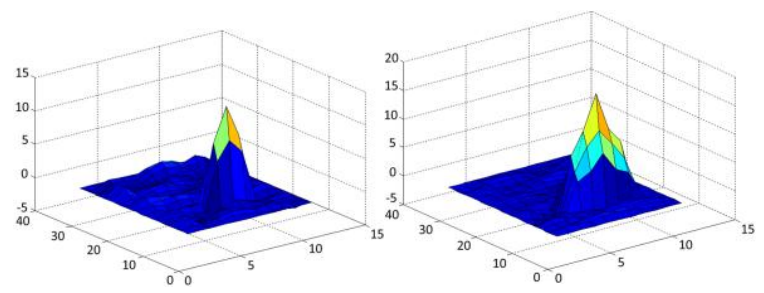

(b)

(c)

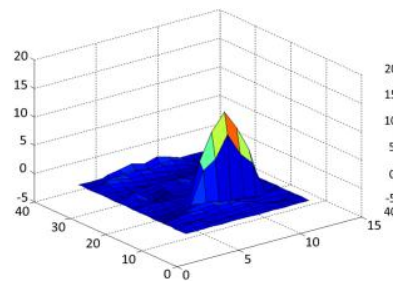

(d)

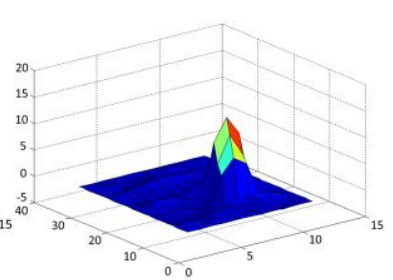

(e)
Figure 6. (a) Original touch signal; (b)-(e) four reconstructed touch signals.

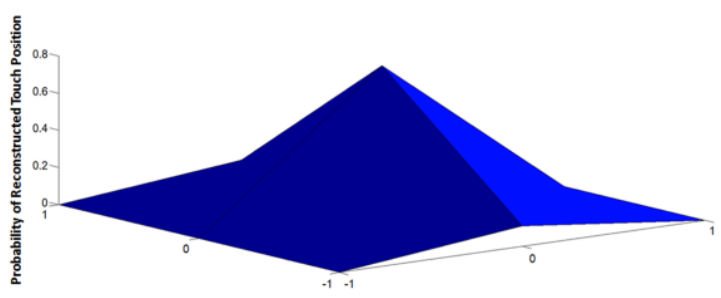

(a)

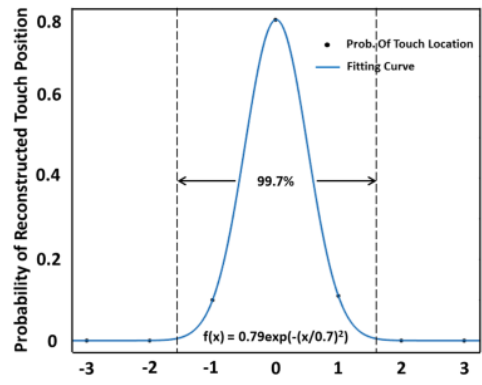

(b)

Figure 7. (a) Distribution of the reconstructed touch positions; $X-Y$ coordinates indicate the distance between the reconstructed touch position and original touch position. (b) The cross-section with the largest variance of (a).

cross section, we can learn that $99.7 \%$ possibility lies between 2 to 2 . Therefore the fast scan radius should be 3 . 


\section{Summary}

In this paper, a force sensing technique and noise reduction techniques are presented for capacitive touch panels. In [1], the deflection caused by the applied force is employed for force sensing. However, when a force load is applied at the edge of a touch panel, the deflection is too small to be measured accurately, resulting in poor force sensitivity. In contrast, enough stress at the edge of the touch panel can be used to interpret the force level. However, additional circuitry for force sensing results in higher power consumption. Thus, downsampling and reconstruction algorithms are proposed to solve this. Force sensing and down-sampling combined techniques yield high accuracy force sensing and low power consumption for capacitive touch screen panels.

\section{Acknowledgements}

Authors thank to the EU-FP7 under Project ORAMA CP-IP 246334-2.

\section{References}

[1] O. Raymundo, "iPhone 6S display teardown reveals how 3D Touch sensors actually work" (2015).

[2] H. Akhtar and R. Kakarala, "A methodology for evaluating accuracy of capacitive touch sensing grid patterns" IEEE/OSA Journal of Display Technology, 10(8), 672-682 (2014).

[3] L. Rieusset and P. Gilles, "Recent developments in the Navier-Stokes problem”, CRC Press (2002). 